NOTAS Y DISCUSIONES

\title{
La recepción de la Teoría Crítica en España
}

\author{
JOSE MARIA MARDONES \\ Instituto de Filosofía, CSIC
}

La Escuela de Francfort comenzó a resonar en España poco después de que comenzara su "redescubrimiento" en Alemania Federal. Los introductores fueron un grupo de profesores y editores españoles que tenian estrechas vinculaciones con el ámbito germano. EI momento coincide con la efervescencia estudiantil que recorrió los países occidentales a mediados de la década de los sesenta.

Entre nosotros, el interés por la Teoría Crítica de la Escuela de Francfort no solamente tenía el sentido de una gran sintonía con las contradicciones culturales del sistema que los francfortianos venían analizando, cada vez con mayor pesimismo, desde los años treinta; aquí, esta referencia cultural estaba marcada por la peculiaridad espanola: la revuelta contra el secuestro de la Ilustración llevada a cabo por el fran. quismo y la búsqueda de un nuevo or. den socio-político que nos igualara al Occidente democrático. Desde este punto de vista, la difusión de la Teoría Crítica en España forma parte de toda una reacción cultural y política por introducir aire fresco en una atmósfera viciada y cerrada.

El movimiento cstudiantil se convirtió en el gran publicista de algunos de los representantes de la Teoría Crítica.
H. Marcuse, sobre todo, gozó de amplio reconocimiento, mucho más allá de los muros académicos. El hombre unidimensional llegó a ser un lugar común del estudiante y del izquierdista de aquellos tiempos.

Pero antes ya se habian hecho presentes, entre los atentos a los ensayos germanos, los nombres de $M$. Horkheimer y T.W. Adorno. A través del impulso editorial de Jesús Aguirre, se comenzaron a traducir en España algunas de las obras fundamentales de Horkheimer y Adorno, y vía Argentina nos llegaron otras obras claves de dichos autores y de Habermas, en la colección de «Estudios Alemanes» admirablemente orientada por E. Garzón Valdés y R. Gutiérrez Girardot. Desde Latinoamérica - Argentina y México-, nos habían introducido profusamente ( $y$, a menudo, sin saberlo) en la lectura de un antiguo miembro de la Escuela de Francfort empeñado en la síntésis entre psicoanálisis y marxismo: E. Fromm. El resultado de esta sensibilidad editora fue que los lectores españoles estuvieron en contacto con la Teoría Crítica de la Escuela de Francfort antes que en Francia y que en el mundo anglosajón. $Y$ aunque la lectura y recepción de esas obras era minoritaria, dado el clima intelectual y el mismo contenido de los textos, 
sin embargo, se fue fraguando entre los cultivadores de las ciencias sociales y la filosofía una serie de referencias y un talante deudor de esta tradición.

Las traducciones prosiguieron hasta casi completar el conjunto de la obra de M. Horkheimer, T.W. Adorno y H. Marcuse. La segunda generación de la Teoría Crítica comenzó a hacer aparición cada vez con más fuerza, como es el caso de J. Habermas y A. Schmidt y, actualmente, es frecuente ver entre las novedades editoriales los ensayos de C. Offe y A. Wellmer, considerados la tercera generación de la Teoría Crítica.

Desde el punto de vista editorial, cabe señalar que desde la transición democrática la atención traductora a los autores de la Teoría Crítica se ha situado casi exclusivamente en España. Madrid y Barcelona son hoy los lugares desde los que se nos pone en contacto, con bastante rapidez, con los autores e ideas de la Teoría Crítica.

Recepciones generales e introducciones

Un primer indicador de la recepción de la Teoria Crítica en España es la presencia de sus principales tesis y autores en los diccionarios filosóficos y de terminología científico-social publicados en las dos últimas décadas. Así, José Ferrater Mora, en su extraordinario y reconocido Diccionario de Filosofia (Madrid, Alianza, 1979; $1 .^{2}$ ed. 1965), incluye en sus artículos la atención a la Escuela de Francfort y a cada uno de sus autores más representativos.

Otro tanto hay que decir del Diccionario de Filosofía contemporánea, compilado por Miguel A. Quintanilla (Salamanca, Sígueme, 1976): recoge las ideas francfortianas fundamentales en breves pero atinados artículos sociales del sociólogo Julio Carabaña. Este interés de los científicos por la Teoría Crítica que- da refrendado en la obra dirigida por Román Reyes, Terminología cientificosocial (Barcelona, Anthropos, 1988), donde de nuevo aparecen entradas dedicadas a la Teoría Crítica y referencias obligadas en artículos de epistemología, filosofía de las ciencias sociales, o en los tratamientos de la modernidad/postmodernidad, etc. Esta presencia de los científicos sociales a la hora de dar cuenta de las ideas de la Teoría Critica en el que fuera el diccionario de filosofía de los «jóvenes filosofos" españoles de aquellos dias, como en las enciclopedias de terminología científico social, marca una tendencia observable en la recepción de la Teoría Crítica en España y fuera de nuestras fronteras: tiene lugar, con tanto o mayor interés, entre los cultivadores de las ciencias sociales como entre los filósofos. El carácter de filosofía mediada por las ciencias sociales, que Horkheimer queria que fuese la Teoría Crítica de los años treinta, ha propiciado este interés compartido por sociólogos y filósofos respecto a la Escuela de Francfort.

Aquí mismo hay una indicación para efectuar una división, aunque sólo sea pedagógica, ya que se pucden presuponer afinidadès filosófico-sociales entre ambos grupos.

Pero antes de entrar en dicho apartado, hagamos una mención acerca de las introducciones o presentaciones de la Teoría Crítica de Francfort en el ámbito español. Una de las primeras que llegó al lector español fue la del italiano G.E. Rusconi, Teoría Crítica de la Sociedad (Barcelona, Martínez Roca, 1969), después le seguirían la del argentino H.C.F. Mansilla (1976) y las de M. Jay, Zima, Perletti... Pero hay que esperar hasta 1985 a que A. Cortina, $\mathrm{Cr}$ tica y Utopia: La Escuela de Frankfurt (Madrid, Cincel, 1985), haga la prime- 
ra introducción española a la Teoría Crítica. Antes de esta presentación, no habían faltado en artículos y prólogos referencias a la historia, vicisitudes y etapas de la Teoría Crítica, pero no se había abordado una introducción o presentación general. Se puede discutir si este trabajo de A. Cortina cumple este requisito. Poseedor de la claridad y brillantez que caracterizan a la autora, adolece, sin embargo, como dice J. Muguerza, su prologuista, de narrar la historia desde el pensamiento de Habermas «apelianamente» interpretado.

\section{La Teoria Crítica en la sociología española}

Las referencias de la Teoría Crítica a la tradición sociológica y su sensibilidad para el dato y las interpretaciones sociales globales, la hacian especialmente atractiva en el clima socio-cultural del tardo-franquismo. En la academia sociológica, en seguida llamó la atención el talante de este análisis de la sociedad. De aquí que fuera la dimensión epistemológica una de las primeras en encontrar eco. La traducción de La disputa del positivismo en la sociología alemana, de TW. Adorno/K. Popper y otros (Barcelona, Grijalbo, 1972), de Jacobo Muñoz, puso en las manos de profesores y estudiantes la denominada "tercera gran disputa metodológica de las ciencias sociales en el ámbito lingüístico alemán». J. Jiménez Blanco, en «Sobre la disputa del positivismo en la sociologia alemana” (en Jiménez Blanco y Moya Valgañon [comps.]: Teoría sociológica contemporánea, Madrid, Tecnos, 1974 [2.a ed. 1978]), abordó la problemática debatida entre teóricos criticos y racionalistas críticos. Al igual que el prologuista y traductor J. Muñoz, Jiménez Blanco se muestra escéptico ante las exageradas virtualidades de «la ra- zón dialéctica». Se inclina más hacia el talante del racionalismo crítico. Tampoco cree en las posibilidades teóricoepistemológicas del marxismo más rigurosamente contemporáneo que propone J. Muñoz. Tanto la traducción como el comentario de Jiménez Blan$c o$, sirvieron para difundir la problemática de la Teoria Crítica, aunque se desconociera $u$ olvidara la sensibilidad "analítica» de la Teoría Crítica de los años treinta y no se vieran en todo su alcance las cuestiones metodológicas y socio-políticas que estaban en juego en «la disputa».

La cuestión de la teoría y el método sociológicos será uno de los problemas que mantendrá la atención de la sociología española. Miguel Beltrán, Ciencia y sociología (Madrid, 1979) utilizará como uno de sus dialogantes a la Escuela de Francfort en la revisión de dicha cuestión por las principales estaciones y autores. Aspectos más concretos, que abordan la polémica Teoria Crítica y hermenéutica, Habermas y Gadamer, son tratados en artículos de Ricardo Montoro (1981); Jordi de Cambra (1982) volverá sobre el enfrentamiento entre Adorno y Popper. El autor de esta reseña también abordó con un fuerte sesgo epistemológico la cuestión de los rasgos característicos de la teoría sociológica de la Teoría Crítica de Max Horkhcimcr. El estudio apareció con el título Dialéctica y sociedad irracional. La Teoria Critica de M. Horkheimer (Bilbao, Univ. Deusto, 1979). Escrito desde el contacto con los estudiantes de sociología, busca dar cuenta, a través de la figura de Horkheimer, de la concepción de Teoría Crítica de la primera generación desde los años treinta a los sesenta. Posee el agarrotamiento de los afanes de concisión y rigurosidad, pero expone con seriedad y crítica el pensamiento del líder de la 
Escuela de Francfort. José E. Rodríguez Ibáñez es el sociólogo español que con más persistencia rastrea y reflexiona sobre el impacto de la Teoría Crítica en la sociología. Posee una trilogía de libros en los que están presentes los nombres y la problemática de los autores francfortianos. El primer título, Teoría Crítica y sociologia (Madrid, Siglo XXI, 1978), reflexiona sobre la tradición crítica de un modo abierto, no reducido a la Escuela de Francfort, y sobre las diversas corrientes que actualmente pudieran cooperar en la formación de una Teoria Crítica de la sociedad. Junto a la fenomenología, el interaccionismo simbólico, el intercambio social generalizado de Lévi-Strauss, aparece la Escuela de Francfort. Se prima al Adorno de la crítica al autoritarismo y al fascismo y al Habermas de la crisis de legitimación del capitalismo tardío.

El segundo estudio, El sueño de la razón. La modernidad y sus paradojas a la luz de la teoria social (Madrid, Taurus, 1982), trata el problema de la racionalidad y la crisis de la modernidad, que repasa desde Kant hasta la Escuela de Francfort pasando por Hegel, Marx y los clásicos de la sociología. $\mathrm{Da}$ cuenta de las diversas dimensiones de la razón y confronta, finalmente, el diagnóstico y solución habermasianos con los del último Parsons y Luhmann. El resultado es favorable al "paradigma comunicativo" de J. Habermas. Se trata de un ensayo que plantea bien las cuestiones, aunque su tratamiento, al ser tan abarcador, no pueda detenerse en la complejidad de los problemas.

Cierra, esperamos que temporalmente, la trilogía de Rodríguez Ibáñez, el reciente trabajo La perspectiva sociológica: historia, teoria y método (Madrid, Taurus, 1989). Es una reflexión epistémica de la tradición sociológica donde la Escuela de Francfort entra en liza con el funcionalismo y sistemismo. Se percibe una mayor distancia del autor respecto a los Adorno, Horkheimer y Habermas. Sobre todo, es ahora más crítico con la teoría de la acción comunicativa de J. Habermas. Acepta las reservas críticas que los Geuss y Boudon le hacen a Habermas y, aunque acepta la riqueza del legado francfortiano, persigue su exploración abierta a otras corrientes y autores con las que revitalizar la autorreflexión sociológica.

La temática de la Escuela de Francfort es cita obligada en los trabajos sociológicos qua analizan algunos aspectos de la tradición marxista. Uno de los que le dedican una de estas referencias de repaso histórico es Emilio Lamo de Espinosa, La teoria de la cosificación: de Marx a la Escuela de Francfort (Madrid, Alianza, 1981). Critica la interpretación de la primera generación sobre las relaciones entre sociedad y trabajo productivo y ve en la alternativa comunicativa sugerida por el Habermas de "conocimiento e interés» una salida, que el mismo Habermas y sus críticos habrian de matizar enormemente.

\section{Filosofia y Teoria Critica}

La Teoría Critica ha resonado ampliamente entre los cultivadores de la filosofía en España en las dos últimas décadas. Aunque el autor con más referencias y glosas sea, sin duda, J. Habermas, no faltan los que poseen en sus origenes un primer contacto, que siguen revitalizando, con la primera generación de los Horkheimer, Adorno y Benjamin. Desde el punto de vista temático, las cuestiones epistemológicas, éticas, políticas y religiosas, son las que atraen mayor número de estudiosos. Veamos, siguiendo esta división, los tratamientos más relevantes. 


\section{Cuestiones epistemológicas}

La fundamentación normativa de la Teoría Critica fue un problema que dejó irresuelto la primera generación de la Escuela de Francfort. J. Habermas se ha hecho eco de esta carencia y ha tratado de darle una respuesta a través del «cambio de paradigma» o paso de la filosofía de la conciencia presa del modelo sujeto-objeto a la teoría de la acción comunicativa o del modelo intersubjetivo. José María Mardones explora en Razón comunicativa y Teoria Critica (Bilbao, Univ. País Vasco, 1985) el intento habermasiano de fundamentar la Teoría Crítica de la sociedad. Tras analizar los dos pivotes de esta construcción, la teoría de la acción comunicativa y la teoría social de la evolución, llega a la conclusión de que este grandioso intento no llega a colmar la radicalidad y universalidad que pretende, velando un concepto de esperanza y solidaridad de raíz teológica, del que fueran mucho más conscientes Horkheimer; Adomo y Benjamin.

Otro aspecto oscuro del intento de Habermas es cl carácter de aciencia reconstructiva" que quiere para su teoría de la acción comunicativa. Manuel Jiménez Redondo, estudioso de Adorno, que se ha convertido en el traductor al castellano de Habermas, analiza estas tensiones entre filosofía y ciencia en su artículo "Filosofía y ciencia reconstructiva" (en J.A. Gimbernat y J.M. González [eds.]: Actas del II Encuentro Hispanoamericano de Filosofia moral y politica, Madrid, Instituto de Filosofía CSIC, 1988). Una hermosa síntesis de las pretensiones y del modo y limitaciones del filosofar de J. Habermas.

\section{Etica y acción comunicativa}

La vertiente de "ética comunicativa" que posce la teoría de la acción haber- masiana ha sido, sin duda, el tema que mayor atención y debate ha suscitado en los filósofos éticos españoles.

Javier Muguerza ha dedicado a Habermas una creciente atención desde que en 1973 analizara la reactualización del concepto de «razón práctica» en el teórico crítico alemán. Si entonces $\mathrm{Ha}$ bermas tenía, a juicio de Muguerza, un pensamiento desiderativo corriendo tras una genérica "emancipación", en su última obra, Desde la perplejidad (Madrid, Fondo de Cultura Económica, 1990), la confrontación es mucho más pormenorizada y atenta a los últimos desarrollos del pensamiento habermasiano. Muguerza sigue siendo crítico con las proclividades de Habermas al cognoscitivismo y a las filosofias hegelianizantes de la historia en forma de teoría de la evolución social, así como a la búsqueda de consenso que, a su juicio, amenaza el disenso y el espíritu crítico que honran a la tradición de la Escuela de Francfort. Resalta, sobre todo, en la obra habermasiana, el ser una excelente continuadora de la tradición de pensamiento que discurre de Rousseau a Marx y en la que corresponde a Kant un lugar central.

Adela Cortina también ha dedicado atención a la ética de la comunicación. $\mathrm{Su}$ interlocutor más directo es K.O. Apel, pero dadas las vinculaciones y afinidades con Habermas, éste es objeto de numerosas referencias en Razón comunicativa y responsabitidad solidaria (Salamanca, Sígueme, 1985) y en Ética minima (Madrid, Tecnos, 1986). El punto en el que discrepa con Apel del intento habermasiano yace en la pretensión "reconstructiva" de la teoría y ética comunicativas. La no exención de contrastaciones empiricas y el rechazo de Habermas de una fundamentación trascendental le parecen a A. Cortina, con Apel, una ingenuidad. Recupera. 
mos, vía ética, los problemas de una fundamentación normativa y del estatus de uciencia reconstructiva" que $\mathrm{Ha}$ bermas busca para su teorización éticosocial.

En este punto de las diferencias Habermas-Apel, han incidido algunos trabajos de Agapito Maestre. Este autor, que inició su vida académica con un estudio sobre T. Adorno y M. Horkheimer, Racionalidad y antropologia negativa (Madrid, Aporía, 1981), ha tratado de aclarar y precisar las relaciones y diferencias entre los dos amigos mediante entrevistas y atinados comentarios críticos. Véase, a título de ejemplo, su Re. flexión para una ética en democracia: discurso ético y utopia (en J.M. González y F. Quesada [coords.]: Teoría de la democracia, Barcelona, Anthropos, 1988).

J. Rubio Carracedo es otro ético en el que Habermas juega un papel de interlocutor. La confrontación con J. Rawls y su «posición original», "Posición original y acción comunicativa" (en Actas del II Encuentro Hispanoamericano de Filosofia moral y politica, ed. cit.), le permite, contra Habermas, acentuar lo que une, antes que lo que separa, a ambos autores. Parecida posición defienden también R. del Águila y F. Vallespín, «La racionalidad dialógica: sobre Rawls y Habermas" (Zona Abierta, 31 [1984], pp. 93-125). Rubio Carracedo dedica también buen número de páginas y referencias a Habermas en su obra $E l$ Hombre y la ética (Barcelona, Anthropos, 1987). Aqui, la confrontación con Habermas se vuelve crítica de la asunción del desarrollo evolutivo de la moral debida a L. Kohlberg.

Crítico de las insuficiencias de la fundamentación racional de la moralidad habermasiana lo es también $E$. Menéndez Ureña, Ética y modernidad (Salamanca, Univ. Pont., 1984). Buen conocedor de Habermas, a quien dedicó una tesis doctoral, es autor de una breve pero clara y vigorosa síntesis de $L a$ Téoría Critica de la sociedad de J. Habermas (Madrid, Tecnos, 1978). Lógicamente, no aborda los últimos desarrollos de la teoría de la acción comunicativa.

Sin poder incluir, igualmente, todo el desarrollo de la teoria comunicativa, Raúl Gabás ha escrito un estudio documentado y riguroso acerca de J. Habermas: dominio técnico y comunidad lingüistica (Barcelona, Ariel, 1980). Una confrontación crítica con el intento habermasiano de fundamentación discursiva de la verdad y la ética desde la sensibilidad conservadora de autores como Spaemann, y Lübbe, Lobkowicz, etc., es la realizada por Daniel Innerarity, $P r a-$ $x$ is e intersubjetividad (Pamplona, Eunsa, 1985). El autor recoge los reparos de estos pensadores al itinerario filosófico habermasiano y duda con ellos de su viabilidad.

\section{Capitalismo, modernidad y postmodernidad}

Habermas ha atraído la atención de los pensadores políticos mediante sus análisis acerca de la invasión de la racionalidad estratégico-instrumental en el ámbito de la política. La traducción parcial de Teoria y praxis (1966) supuso un impulso que se vio fortalecido por Problemas de legitimación en el capitalismo tardio (1975). Comentaristas y glosadores de estos temas han sido, entre nosotros, E. Menéndez Ureña, José María González y el que esto escribe. Hay que agradecer a Antoni Domènech la traducción (no el cambio de titulo) de Strukturwandel der Öffentlichkeit (Historia y crítica de la opinión pública, Barcelona, Gustavo Gili, 1981), - con un prólogo en el que deja claras, sus distancias respecto a Habermas y su peculiar neo-marxismo. 
En el campo del pensamiento políti$c o$, quien está obteniendo creciente eco y atención es el politólogo, discípulo de Habermas, Clauss Offe. Uno de los primeros en divulgar críticamente el pensamiento de Offe ha sido el filósofo del derecho Elías Díaz. En su obra, De la maldad estatal y la soberania popular (Madrid, Debate, 1984), E. Diaz aborda las aportaciones y limitaciones del "nuevo paradigma" político de los nuevos movimientos sociales propuesto por Offe. En el artículo «Socialismo democrático: instituciones políticas y movimientos sociales" (en Sociedad civil y Estado, Madrid, Fundación F. Ebert, 1988), y tras los avances y reflexiones de Offe, el acuerdo acerca del rol de los nuevos movimientos sociales y las instituciones juridico-políticas es mucho mayor.

La primacía de Habermas como representante actual más cualificado de la Escuela de Francfort vuelve a ponerse de manifiesto en el tema de la crisis de la modernidad y el denominado debate de la postmodernidad. Numerosos artículos de ocasión han tocado el tema. Pero, con más seriedad, haciéndose eco de sus confrontaciones con Rorty, Lyotard, etc., estarian algunos artículos como los de M. Jiménez Redondo, "La modernidad, ¿un proyecto inacabado o un proyecto superado? s; R. del Aguila y F. Vallespín, " ¿Necesitamos un punto arquimédico? Teoría Crítica y praxis política " (Congreso sobre Teoría de la Acción y Comunicación, Madrid, 1987); Carlos Thiebaut, «De la ética y el presente" (La balsa de la medusa, 2 [1987], pp. 51-77); J.M. Mardones, «El conservadurismo de los postmodernos" (en Sobre la postmodernidad, Bilbao, Colegio de Doct. y Licenciados, 1988). El debate postmoderno ha servido para presentar y airear las ideas de A. Wellmer (Teoría Crítica de la socie- dad y positivismo, Barcelona, Ariel, 1979). Los análisis y matices de Wellmer respecto a Habermas y su tratamiento de la estética son importantes para este diálogo.

El área de la estética en relación con la Teoría Crítica tiene en Gerard Vilar uno de sus cultivadores más atentos. Adorno y Habermas son los autores sometidos a su consideración y diálogo.

Agapito Maestre es el autor español que se ha hecho eco, con mayor asiduidad, en artículos de opinión, del debate de Habermas con los llamados nuevos historiadores alemanes y con Heidegger.

\section{Filosofia de la religión y Teoría Critica}

Las cuestiones teológicas siempre estuvieron rondando el pensamiento de la primera generación de la Escuela de Francfort. No en vano Horkheimer y Adorno llegaron a afirmar la presencia de una "teología oculta» en la Teoría Crítica.

La mejor confrontación con la filosofía de la religión subyacente al pensa. miento de M. Horkheimer es la de J.J. Sánchez, Wider die Logik der Geschichte (Einsieldeln, 1980), lastimosamente todavía no editada en español.

También J.A. Estrada le ha dedicado atención al tema de la trascendencia en M. Horkheimer, "El Dios de un ateo, la trascendencia en M. Horkheimer» (Estudios Eclesiásticos, 231 [1984], pp. 397-429).

J.M. Mardones dedicó el estudio Teologia e ideologia (Bilbao, Univ. Deusto, 1979) a la confrontación entre la teolo. gía política de la esperanza y el concepto de ideología tomado de los Horkheimer y Adorno. Pretendia obtener, con la ayuda de la Teoría Crítica, indicaciones para un discurso teológico no ideológico. Con una sensibilidad que tiene más 
en cuenta el concepto de razón comunicativa de Habermas y Wellmer, ha escrito el ensayo Postmodernidad y cristianismo (Santander, Sal Terrae, 1988), una confrontación con los pensadores y el talante de la postmodernidad desde sus desafíos al cristianismo.

Concluimos este apartado sobre la recepción de la Teoría Crítica en el pensamiento filosófico-religioso español citando el estudio, no publicado, de M. Reyes Mate sobre W. Benjamin ( Modelos históricos de relación de la religión con la razón"), donde se avanza la hipótesis de que los sujetos de la historia son los sin-sujetos. La «memoria passionis" pasa a ser así una categoría fundamental de la filosofía de la historia. Los escritos posteriores de Reyes Mate, confrontándose con Hegel y la ética comunicativa de J. Habermas, esgrimen criticamente esta categoría. Véase, por ejemplo, "Historia de la libertad y memoria passionis" [Enrahonar, 2 [1981], pp. 87-97) y «Por una ética compasiva» (Razón y Fe, sept.-oct. [1988], pp. 209-216). J.A. Gimbernat, procedente de la órbita de $\mathrm{E}$. Bloch - que dirigió varios años un seminario sobre la Teoría Crítica en el Instituto Fe y Secularidad-, también está ocupándose de las relaciones entre crisis de la modernidad, racionalidad y religión en la Escuela de Francfort.
El recorrido efectuado por autores españoles, estudiosos y ocupados en la Teoría Crítica de la Escuela de Francfort, ha querido mostrar, al menos, por dónde han discurrido estas preocupaciones en cuanto se reflejan y constatan en escritos.

A tenor de lo expuesto, podemos concluir sin exageración que tal influencia no ha sido superficial. Son numerosos los tocados por los problemas de la filosofía crítica. Para algunos es el mismo talante de pensamiento o itinerario filosófico el que se quisiera continuar. Muchos más son lo que los tienen como interlocutores válidos a la hora de plantear o discutir diagnósticos, debatir cuestiones y aventurar soluciones. La Teoria Crítica se constituye así en un venero de impulsos y sugerencias, de contrastaciones y de ocasión para la reflexión más personal. En un momento de revisiones y de exploración de caminos, la referencia a la Escuela de Francfort es una fuente inagotable. La atracción despertada en el tardo-franquismo por un estilo de pensamiento crítico, móvil, atento a los desplazamientos de la realidad y con pasión por la justicia y la libertad, continúa siendo todavía hoy, en los años de la democracia desencantada y del neo-conservadurismo, un acicate para la resistencia y la pugna por la emancipación de toda la sociedad. 